\title{
Le marketing vert comme entrée à l'incarnation de la responsabilité sociale des entreprises
}

\section{Green Marketing as an Entry to the Embodiment of Corporate Social Responsibility}

\begin{tabular}{|c|}
\hline Ouahiba ouldbrahim \\
\hline o.ouldbrahim@univ-boumerdes.dz \\
\hline Universite m'hamed bougera boumerdes (Algerie) \\
\hline
\end{tabular}

\section{Résumé:}

Cet article vise à établir comment marketing vert et responsabilité sociale des entreprises peuvent fonctionner ensemble, en montrant l'importance pour l'entreprise d'adopter une démarche de marketing vert, en intégrant les pratiques RSE dans la stratégie de ce marketing et cela dans le cadre de répondre aux besoins des consommateurs après avoir eu un impact positif sur leurs préférences et commander des produits respectueux de l'environnement, et réduire au minimum les risques environnementaux en respectant et en préservant les lois relatives à la protection de l'environnement.

Mots clés: marketing vert, responsabilité sociale, développement durable, produit vert, parties prenantes.

\section{Code Jel:M14, M31}

\section{Abstract:}

This article aims to establish how Green Marketing and Corporate Social Responsibility can work together, showing the importance for the company to adopt a Green Marketing approach, integrating CSR practices into the strategy of this marketing and this within the framework of meeting the needs of consumers after having a positive impact on their preferences and ordering environmentally friendly products, and minimize environmental risks by respecting and preserving environmental protection laws.

Key words:green marketing, social responsibility, sustainable development, green product, stakeholders.

JEL Classification Codes:M14, M31. 


\section{Introduction:}

À l'heure actuelle, les entreprises sont confrontées à de nombreux défis technologiques, économiques et surtout environnementaux qui les ont amenées à repenser et à s'intéresser davantage à leur culture organisationnel et à accorder une attention particulière au thème de l'intégration de l'environnement dans sa gestion, et répondre aux besoins et aux exigences de la société pour s'assurer que les objectifs en charge sont atteints et surtout qu'elles restent sur le marché, les obligeant à en adopter plusieurs entrées, y compris un marketing attentif à l'environnement qu'on appelle le marketing vert, qui est l'une des entrées modernes au marketing,qui doit mettre sur le marché des produits verts et éthiques, mais pas seulement, il doit travailler sur l'acceptation de ces produits et sur le comportement des individus au moment de l'usage des produits.

Dans cet ordre d'idées, le marketing vert incarne le concept de responsabilité Social, en réduisant les risques environnementaux et en fournissant des produits sûrs au consommateur, obtenant ainsi une performance marketing pour l'entreprise d'un côté, et l'équilibre environnemental de l'autre.

Sur la base de ce qui précède, la problématique de cette recherche peut être développée dans la question fondamentale suivante :

Comment le marketing vert peut-il refléter la responsabilité sociale au sein d'une entreprise?

Et pour répondre à cette question principale, elle a été divisée en sous-questions suivantes :

Qu'entendons-nous par marketing vert? Et qu'est-ce que cela a à voir avec la responsabilité sociale des entreprises?

Afin de répondre aux questions ci-dessus, les hypothèses suivantes ont été testées :

- Le marketing vert s'avère être un phénomène complexe qui veille à créer des actions responsables en instaurant des bonnes pratiques professionnelles. ;

- Le marketing vert peut être source d'efficacité et d'efficience pour la responsabilité sociale des entreprises.

Le présent article a pour but de faire un rapprochement entre le marketing vert et la responsabilité sociale des entreprises et plus spécialement, d'analyser les situations d'une meilleure mutualité entre les deux concepts.

Notre travail s'appuie sur une méthode descriptive et analytique en même temps :

- le volet descriptif englobe la plus grande partie du travail.

- le volet analytique est situé dans la dernière partie. 
Dans le cadre de cet article, nous essayons de répondre à cette problématique en abordant les trois éléments fondamentaux suivants:

I. Le concept du marketing vert et ses fondations.

II. Le concept de la responsabilité sociale des entreprises.

III. Le rôle que joue le marketing vert pour promouvoir la responsabilité sociale -cas de quelques entreprises.

\section{Le concept du marketing vert et ses fondations}

Le concept du marketing vert a fait son apparition à la fin du siècle dernier pour occuper une place importante parmi les chercheurs et ceux qui s'intéressent à l'environnement. Dans cette partie on va présenter l'émergence du concept de marketing vert, sa définition et son évolution.

\section{1 l'historique (le développement) du marketing vert}

Depuis quelques années l'adjectif «vert» est apparu pour qualifier aussi bien le management, que le marketing (MALAVAL, BENAROYA ,2013). ce terme est né avec l'émergence des premiers signes de l'intégration de l'environnement dans les stratégies de marketing des entreprises (premiers emballages recyclables, les poudres à lessive sans phosphate, consommation d'essence réduite pour les voitures, etc.)(www.etudier.com/dissertation/le-marketing-vert), et vers la fin des années 90, plusieurs évènements ont donné naissance à un nouveau type de marketing appelé le marketing vert basé sur des préoccupations environnementales telle que :La pollution, le réchauffement climatique, les avancées technologiques et la réduction des ressources (REMOUSSE, 2005).

Donc, les débuts du marketing vert étaient basés sur le fait que les entreprises ont introduit les questions d'environnement dans leurs stratégies, politiques et leurs processus de production.

\section{I.2 définitions du marketing vert}

Le marketing vert est un phénomène complexe qui a été étudié par le passé, mais dont les définitions et les éléments centraux qui les caractérisent varient d'un auteur à l'autre (BERGERON, 2011), et parmi ces auteurs on trouve :

LAVILLE et DEVEAU qui considèrent le marketing vert comme une stratégie marketing dont les méthodes de fabrication et de commercialisation sont plus respectueuses de

\begin{tabular}{|ll|l|l|}
\hline Volume:04 & $\mathbf{N}^{\circ}: 01$ & Année:2021 & P: 486 \\
& & &
\end{tabular}


l'environnement, et qu'il remet en cause les fondements des quatre « $\mathrm{P} »$ (DE GERUS, 2013).

D'après Fernandez SAUVEUR, qui propose une définition générale, le « marketing vert a pour but la conception, la fabrication, la distribution et la promotion des produits et services commerciaux qui, au-delà de qualités économiques et d'une satisfaction individuelle, soient aussi socialement et environnementalement responsables » (REMOUSSE, 2005).

Michael Jay POLONSKY a plaidé en faveur de la définition suivante: « Le marketing vert consiste en toutes les activités désignées à générer et faciliter tout échange destiné à satisfaire les besoins et désirs humains ; cette satisfaction se produit avec le minimum d'impact détriment sur l'environnement naturel »(MILED, FARHANI, 2012).

Pour sa part, Le Gall prône l'idée que le marketing vert reste « limité à un nombre limité d'entreprises qui satisfont les besoins d'un nombre limité de consommateur verts en offrant un nombre limité de produits verts ». L'auteur suppose donc que le marketing vert traditionnel ne semble plus adapté aux orientations du marché (DE GERUS, 2013).

Apres avoir analysé ces diverses définitions du concept, on peut conclure que le marketing vert est une stratégie qui se base sur des caractéristiques écologiques d'un produit, sur des promotions verte, sur des promesses environnementales, et sur la valorisation du consommateur qui double son acte d'achat d'un acte en faveur de la nature.

Donc, le marketing vert est concentré sur la conception et promotion des biens et services qui contribuent à une valeur ajoutée écologique à une au minimum des étapes de leur cycle de vie (production respectueuse de l'environnement ou commerce juste des matières premières, processus de fabrication propre, emballage réduit, récupération et recyclage, ...etc.)(LAVILLE, 2009).

\section{3 Trois notions à distinguer (concepts voisins)}

Ils sont apparues de nombreux concepts se rapprochant du marketing vert s'intéressant à la combinaison du marketing et de l'environnement, c'est le cas du: marketing environnemental, écologique, responsable, durable» (MILED, FARHANI, 2012).

- Marketing environnementale: il consiste à prendre en considération toutes les pratiques pouvant engendrer et faciliter n'importe quel type d'échange ayant pour but de satisfaire les désirs et les besoins humains, de telle sorte que la satisfaction de ces besoins et désirs ait lieu avec un impact négatif minimal sur l'environnement naturel (DE GERUS, 2013). 
- Marketing écologique: Selon Stanton et Futrell le marketing écologique est constitué de l'ensemble des actions qui vise à développer un positionnement écologique d'une marque ou d'un produit pour améliorer l'image de l'entreprise et augmenter les ventes (MILED, FARHANI, 2012).Ce type de marketing peut se baser sur les caractéristiques écologiques d'un produit (matières premières écologiques, produit recyclable ou biodégradable,..), sur des promotions vertes (arbre planté pour un achat) ou sur les promesses environnementales de l'entreprise (fondation, actions écologiques) (COUTURIER,2014).

- Marketing durable:Van Dam et Apeldoorn introduisent le concept de marketing durable, qui est le marketing pilier d'un développement économique durable. Le marketing durable requiert de trouver des cadres de régulation optimaux pour gouverner le rôle du marketing dans un espace écologique limité (MILED, FARHANI, 2012).

\section{4 Importance du marketing vert:}

Le marketing vert puise sa raison d'être, d'abord à une détermination des responsables de marketing de se conformer aux nouvelles revendications environnementales des consommateurs, ensuite à leur prise de conscience des atteintes causés à l'environnement (MILED, FARHANI, 2012).

Il présente certains avantages qui sont bénéfiques pour les entreprises car il permet d'augmenter le sentiment d'appartenance des employés, de se démarquer de la concurrence, d'augmenter les parts de marché (les consommateurs BIO sont prêts à payer cher plus que les autres), de fédérer les individus (Nous sommes unis pour une même cause) et d'obtenir un avantage concurrentiel.

Donc, le marketing vert est important pour divers groupes :( REDJEF, ARABA, 2018)

- pour les pouvoirs publics car ils se voient la nécessité de formuler des lois et des réglementations concernant la protection de l'environnement.

- pour les entreprises car elles redoivent produire et distribuer en tenant compte du facteur environnemental.

- pour les consommateurs qui supportent les décisions des pouvoirs publics et des entreprises, mais qui à travers leurs comportements et décisions individuels peuvent encourager la protection de l'environnement car ils exercent un poids important lors du choix des produits.

\section{II. le concept de la responsabilité sociale des entreprises}

\begin{tabular}{c|c|cc|c|c|}
\hline Revue internationale de la performance économique & Volume:04 & $\mathbf{N}^{\circ}: 01$ & Année:2021 & P: 488 \\
ISSN: 2661-7161 EISSN:2716-9073 & & & &
\end{tabular}


Le concept de la responsabilité sociale des entreprises est apparue dans les années 1950 et depuis il s'est profondément muté selon les conditions de son émergence. Cette partie de l'article présente l'émergence de ce concept, son évolution et sa définition.

\section{1 les phases de développement de la RSE :}

La compréhension du concept de la responsabilité sociale a traversé de nombreuses étapes historiques : (CHERKAOUI, 2015).

1. Années 1950 et 1960 : Cette phase s'est caractérisée par la domination des enjeux lier à la définition du concept RSE ainsi que ses fondements éthiques et économiques. Dans une telle période, nous avons assisté à l'apparition d'une approche législative et philosophique de la RSE.

2. Années 1970 : cette transition s'est caractérisée par l'augmentation des courants sociaux et environnementaux sollicitant l'entreprise. Les travaux de recherche sur la RSE ont pris une approche pragmatique et managériale, telle que es travaux d'Ackerman et Bauer (1976) qui ont été à l'origine du l'introduction de la vision de la "prise de conscience ou sensibilité sociale de l'entreprise". Il s'agit de la gestion de la responsabilité sociale des entreprises et de la diffusion et de la mise en œuvre des pratiques de RSE

3. Années 1980-2000 : dans cette phase une nouvelle définition est apparue remplaçant celle de la réactivité sociale de l'entreprise. Il s'agit de la performance sociétale des entreprises (PSE) (traduit $\mathrm{du}$ "Corporte Social Performance"). Le concept de PSE représente alors un aperçu des deux précédentes approches.Il se concentre sur les capacités de gestion de la responsabilité sociale des entreprises, ainsi que sur l'impact et la mesure des politiques de responsabilité sociale des entreprises.

\section{2 définition de la responsabilité sociale :}

Le concept de responsabilité sociale des entreprises a fait son entrée dans le débat international vers la fin des années 90, et plusieurs définitions sont attribuées à ce concept notamment :

La Commission européenne définit la RSE comme " l'intégration volontaire par les entreprises des préoccupations sociales et environnementales à leurs activités commerciales et leurs relations avec les parties prenantes » (STOKKIN, NOTAT, 2010).

Une autre conception est donnée à La responsabilité sociale de l'entreprise par Bowen repose sur le concept de «contrat social » entre l'entreprise et ses parties prenantes directes (actionnaires, salariés, fournisseurs, clients...) et indirectes (administrations, collectivités locales, groupes d'intérêt, vecteurs d'opinion, société civile...) (SI TAHAR, MEZIANI, 2018).

\begin{tabular}{|ll|l|l|}
\hline Volume:04 & $\mathrm{N}^{\circ}: 01$ & Année:2021 & $\mathrm{P}: 489$ \\
& & &
\end{tabular}


Selon le WBCSD (The World Business Council for SustainableDevelopment), la RSE est « l'engagement continu des entreprises à agir correctement sur le plan de l'éthique et de contribuer au développement économique, tout en améliorant la qualité de vie de ses employés et de leurs familles, de la collectivité locale et de l'ensemble de la société ».

Même dans cette formulation, la RSE est considérée comme une des trois responsabilités clés des entreprises, y compris la responsabilité économique et la responsabilité environnementale (RODIC, 2007).

L'Organisation de coopération et de développement économiques (OCDE) propose une définition descriptive en estimant que la RSE peut signifier la contribution des entreprises au développement de la durabilité; que le comportement des entreprises doit non seulement assurer des dividendes aux actionnaires, des salaires aux employés et des produits et services aux consommateurs, mais il doit répondre également aux préoccupations et aux valeurs de la société et de l'environnement(RODIC, 2007).

L'ISO 26000 donne la définition suivante de la RSE : la responsabilité d'une organisation vis-à-vis des impacts de ses décisions et activités sur la société et sur l'environnement, se traduisant par un comportement éthique et transparent qui prend en compte les attentes des parties prenantes (OUBBA, AKRICH, 2018).

Donc, d'après les précédentes définitions on conclu que la responsabilité sociale des entreprises repose sur trois critères, à savoir :

Environnemental (compatibilité entre l'activité de l'entreprise et le maintien des écosystèmes), social (conséquences sociales de l'activité de l'entreprise) et économique (performance financière).

\section{3 Les piliers de la responsabilité sociale des entreprises}

La responsabilité sociale des entreprises se base sur la jonction de trois grands piliers fondamentaux que nous proposons de reprendre en détail.

- Le pilier économique : une entreprise socialement responsable soutien l'économie locale en se fournissant auprès d'organismes locaux dans la mesure du possible et en investissant dans la région. Elle fait aussi preuve de transparence auprès de ses investisseurs et de ses clients (www.caderemloi.fr). Autrement dit il faut :

- $\quad$ satisfaire et fidéliser le client ;

- $\quad$ soutenir les fournisseurs locaux ;

- $\quad$ payer les factures dans les délais.

- Le pilier social : c'est l'un des aspects les plus importants de la responsabilité sociale des entreprises et parmi ses priorités on trouve :(www.generali.fr)

- le renforcement de l'égalité des chances et de la diversité ;

\begin{tabular}{|ll|l|l|} 
Volume:04 & $\mathbf{N}^{\circ}: 01$ & Année:2021 & P: 490 \\
& & & \\
& & &
\end{tabular}


- la garantie de l'hygiène et de la sécurité sur le lieu de travail ;

- le respect des droits des travailleurs ;

- et la formation du personnel.

- Le pilier environnemental: qui se manifeste par l'utilisation minimale des ressources épuisables telles que le pétrole et le gaz, l'utilisation durable des ressources renouvelables et la protection de la capacité de prise en charge de la nature avec le souci de préserver les ressources naturelles et favoriser la diversité biologique, sans oublier de réduire l'émission des gaz à effets de serre et de limiter les déchets, notamment ceux qui sont dangereux .

\section{4 Les différentes approches théoriques de la responsabilité sociale des entreprises}

Les fondements théoriques de la responsabilité sociale d'entreprise RSE reposent sur une double vision :

Dans la première vision s'inscrivent l'approche néo-classique, la théorie de l'agence, et dans la deuxième vision la théorie de parties prenantes et les théories néoinstitutionnelles.

- Théorienéo-classique :Selon l'approche néo-classique de la RSE, dont le principal auteur est Milton Friedman, la responsabilité sociale de l'entreprise est d'accroître ses profits au profit des actionnaires,c'est-à-dire que les affaires doivent être essentielles voire les seules responsables dans la création de profits à ses propriétaires(RODIC, 2007), et cela dans la mesure où elle respecte les règles du jeu, c'est-à-dire celles d'une concurrence ouverte et libre.

- Théorie de l'agence : Cette théorie se propose de définir les conditions d'un contrôle des dirigeants par les actionnaires. Et d'après cette approche, les dirigeants ne sont que des mandataires des actionnaires. Ils ont des responsabilités envers les propriétaires qui les emploient dans le sens où ils doivent mener le travail comme ceux-ci le désirent (CHAKOR ,DINIA, 2018)

- Théorie des parties prenantes:Depuis les années 1970, l'expression « stakeholders» est utilisée dans les théories de management et de gestion. Dans sa publication «Strategic management : astakeholder approach», Edward Freeman définit les parties prenantes comme " tout groupe ou individu qui peut être affecté ou est affecté par l'accomplissement des objectifs d'une organisation », cette théorie établit que la nature des parties prenantes d'une organisation, leurs valeurs, leur influence

\begin{tabular}{|ll|l|l|}
\hline Volume:04 & $\mathbf{N}^{\circ}: 01$ & Année:2021 & P: 491 \\
& & &
\end{tabular}


relative sur les décisions et le type de situation constituent des informations relevantes pour prévoir le comportement organisationnel(NOTAT, STOKKINK,2010).

Cette approche place l'entreprise au centre d'un ensemble d'acteurs (les parties prenantes, stakeholders) qui ont des attentes implicites ou explicites à son égard, qui ont un pouvoir plus ou moins fort pour se faire entendre et influencer les dirigeants (QUAIREL, CAPRON, 2013).

- Théorie néo-institutionnelle :Pour la Théorie Néo-Institutionnelle (TNI), notamment développée par W. DiMaggio et P. Powell, est une théorie de l'environnement des organisations, qui propose d'analyser les raisons institutionnelles et symboliques qui expliquent l'adoption de pratiques de gestion identiques dans les organisations (OUBBA, AKRICH, 2018).

Ce courant théorique considère que les organisations au départ mécanistes (créées pour atteindre des objectifs d'ordre technique) ont une tendance à se transformer progressivement en institutions. Mais pour le néo-institutionnalisme, ce sont bien certains acteurs (entrepreneurs institutionnels, parties prenantes) qui, dans la pratique, jouent avec les règles institutionnelles et peuvent alors réussir à légitimer des changements dans un champ institutionnel (DIMAGGIO, POWEL,www.sietmanagement.fr).

\section{5 Mise en œuvre de la RSE au niveau des entreprises}

Pour mettre en place une démarche RSE dans une entreprise il faut suivre les étapes suivantes : (NOTAT, STOKKINK,2010)

- Intégration volontaire:La démarche volontaire signifie que l'entreprise est libre de prendre des engagements afin d'améliorer ses performances sociales et environnementales.

-Aller au-delà des obligations légales : l'entreprise doit dépasser les exigences légales en ce qui concerne l'adoption de la RSE.

- Introduire la RSE dans le mode de gestion de l'entreprise : la participation de la haute administration est nécessaire afin de pouvoir bénéficier des ressources financières et matérielles permettant l'adjonction de la RSE.

- Favoriser Le dialogue avec toutes les parties prenantes : pour mettre en place une RSE, il faut qu'il y est un réel gouvernement d'entreprise qui permet de créer des règles et des pratiques concernant les relations entre les différentes parties prenantes,les écouter et prendre en compte leurs avis

\begin{tabular}{|ll|l|l|} 
Volume:04 & $\mathrm{N}^{\circ}: 01$ & Année:2021 & $\mathrm{P}: 492$ \\
& & &
\end{tabular}


- Aller au delà de la recherche de profits économiques : La responsabilité sociale des entreprises ne sert pas qu'à des fins économiques et à maximiser les profits pour les actionnaires, cela ne doit pas être son seul objectif.

- S'adapter au changement : l'entreprise doit s'accommoder aux différents changements, et doit être plutôt dans une dynamique de progrès et d'amélioration continue afin de progresser avec la société.

- L'intégration des préoccupations sociales:l'entreprise doit s'assigner d'introduire les préoccupations sociales aussi bien en interne qu'en externe.

- L'intégration des préoccupations environnementales: dans le cadre de la protection de la planète, l'entreprise doit réduire tous les effets indésirables et négatifs de ses activités, et promouvoir un comportement respectueux de l'environnement.

- L'évaluation de la politique de la RSE : enfin, l'entreprise doit démontrer son comportement responsable par des faits qui sont illustrés par les outils suivants :les chartes, les codes de conduite, les labels et les rapports sociétaux, bilans RSE.

III. Le rôle que joue le marketing vert pour promouvoir la responsabilité sociale -cas de quelques entreprises-

Aujourd'hui, les entreprises essaient de développer des formes de marketing mettant en vue leurs comportements socialement responsables, c'est pour cela nous allons nous intéresser à l'importance que peut porter la RSE pour la fonction marketing (CHAUZAL, 2007).

\section{1 Intégrer la RSE dans les stratégies marketing :}

L'intégration des préoccupations sociales et environnementales dans la définition de la stratégie de l'entreprise devient une nécessité pour renforcer la légitimité de la direction marketing lorsqu'elle décide de s'engager sur ce chemin(FAHD,2013), et pendant longtemps le marketing est mis à l'écart des enjeux concernant la responsabilité sociale des entreprises qui se concentrait plus particulièrement sur les pragmatismes de gestion (approvisionnement, production, gestion de ressources humaines, finance et comptabilité) car le marketing, à travers ses pratiques, a pour objectif de stimuler et d'influencer la demande des consommateurs afin de la faire coïncider avec les objectifs de l'entreprise, et depuis quelque temps on le trouve au milieux des défis de développement durable et de la responsabilité sociale des entreprises(FAHD,2013).

L'idée d'impliquer davantage la fonction marketing autour d'enjeux de développement durable ne peut se réaliser que si l'entreprise concernée est ouverte, dans sa culture professionnelle et dans ses orientations stratégiques, à une prise en compte et à une 
intégration de ces enjeux dans la raison d'être et dans le développement de l'entreprise elle-même (LOUPPE, 2006).

L'entreprise doit avant toute chose développer une forme de marketing qui mit en évidence son comportement socialement responsable. Ce nouveau point de vue consiste à réinventer le marketing et de le rendre plus responsable en le fondant sur les « $\mathrm{P} » \mathrm{du}$ développement durable: les personnes, la planète, les profits et le progrès de l'humanité (BENACEUR, 2016).

L'intégration de la responsabilité sociale par le marketing est recommandable par :(LOUPPE, 2006)

- Une diversification des produits et services faisant apparaître une orientation responsabilité sociale ;

- Le développement de nouveaux produits et de procédés «haute performance»ou «produits propres»;

- Des plans de réduction des déchets et des rejets (organisés par sites de production ou par ligne de produits...) ;

- Le développement de services et de conseils aux clients, prescripteurs et distributeurs orientés «développement durable et responsabilité sociale » (ex bases de données, guide de bonnes pratiques, hot line dédiée...) ;

- La formation du personnel aux enjeux environnementaux, sociaux ou aux risques sanitaires ;

En fait le marketing socialement responsable doit essayer de modifier différentes choses tel que les réactions et le comportement des gens, la manière dont ils perçoivent leur propre image, les relations qu'ils nouent les uns avec les autres, leurs habitudes et leurs valeurs (CHAUZAL, 2007).

En bref, le marketing dans une société durable doit trouver un moyen de maintenir l'équilibre entre l'économie, la société et l'environnement.

\section{2 Relation entre marketing vert et RSE :}

La relation qui existe entre la responsabilité sociale de l'entreprise et le marketing vert n'est pas une relation dans laquelle un nouveau concept remplace un concept précédent, car le concept du marketing et le marketing vert plus spécialement est le domaine vital dans lequel l'entreprise exerce un rôle social et éthique à l'égard de la société.

Il ya un éventail de questions de marketing vert importantes, qui doivent être prises en compte en matière de la responsabilité sociale tel que pratiques de vente, droits

\begin{tabular}{|ll|l|l|}
\hline Volume:04 & $\mathbf{N}^{\circ}: 01$ & Année:2021 & P: 494 \\
& & &
\end{tabular}


des consommateurs, protection de l'environnement et la sécurité des produits (MAIGNAN, 2005).

Le challenge pour l'entreprise qui veut concevoir des produits verts et suivre les démarches de la responsabilité sociale des entreprises est qu'elle doit continuer à produire des biens de qualité identique, si ce n'est supérieur, en consommant moins de ressources et réduire les impacts (économiques, environnementaux et sociétaux) à tous les niveaux du cycle de vie du produit, de l'approvisionnement en matières premières jusqu'à la destruction du produit (MRANI, 2009).

Les entreprises mettent en avant dans leur démarche marketing des actions RSE. Et les facteurs clés du succès du marketing vert à incarner la responsabilité sociale des entreprises sont les suivants :(MRANI, 2009)

- L'engagement ne doit pas être un coup de publicité. Il doit être inscrit dans la culture et la mission de l'entreprise et surtout, être intégré dans les systèmes de management.

- L'engagement doit être simple, transparent, et surtout cohérent avec le marché et le métier de l'entreprise.

- L'engagement doit être exprimé par des actes, plus que par des paroles.

- L'engagement doit s'exprimer dans les moindres détails des actions de l'entreprise: packaging, supports de communication, site web, promotions, ...

- La communication doit être honnête et respectueuse.

- Il faut instaurer une relation honnête et authentique avec les clients et les parties prenantes.

Il existe un certain nombre de situations rencontrées dans la vie quotidienne des consommateurs qui imposent une corrélation d'acte entre marketing et responsabilité sociale des entreprises.

- L'image : Beaucoup d'entreprises aperçoivent la réalité des défis du développement durable lorsque leur image se trouve fragilisé par une contre-performance qui concerne leur mode de gestion.

Des pratiques peu agréables, ou une conduite jugée brusque en matière de la préservation de l'environnement, ou bien au plan de la gestion des ressources humaines et du progrès social peu nuire à la réputation et à l'image d'une entreprise ou d'une marque(LOUPPE, 2006).

- Les produits:Bien que ce soit complexe de parler véritablement de marque sociétale on peut que se rendre compte de l'intégration de la dimension environnementale en marketing.

\begin{tabular}{|ll|l|l} 
Volume:04 & $\mathrm{N}^{\circ}: 01$ & Année:2021 & $\mathrm{P}: 495$ \\
& & & \\
& & &
\end{tabular}


Néanmoins, pour répondre aux problèmes environnementaux, le marketing doit se centrer sur l'amélioration de l'éco-performance des produits mis sur le marché (TCHAM, 2017).

Cela dit que les produits doivent être conçus de façon que l'entreprise prenne en considération les critères environnementaux ou sociaux, en réduisant les impacts négatifs sur l'environnement et sur l'homme qui sont évalué par l'analyse du cycle de vie du produit.

- Le prix:La responsabilité sociale, est appliquée par une politique institutionnelle de prix qui doit être juste, et équilibre entre le coût et le bénéfice réalisé par l'entreprise.

Facturer au consommateur des charges dont il n'est pas responsable ou qui n'ont rien à voir avec le produit ou le service qui lui est rendu, pourra amener, par exemple, à des prix perçus comme «injustifiés»(LOUPPE, 2006).

Il existe un ensemble de lignes directrices qui aident à adopter une politique de prix vert qui comprend une application de la responsabilité sociale:

- Respect des lois sur la concurrence équitables et des mécanismes du marché ;

- Dans la tarification, les segments sociaux doivent être pris en compte ;

- S'engager dans le processus de clarification du prix réel, en indiquant s'il existe une taxe de vente à laquelle le produit est soumis afin que le consommateur ait connaissance du prix réel (LOUPPE, 2006).

- La distribution:La distribution physique du produit est censée être fondée sur un cadre éthique et sur la valeur.

- Transport de produits par des moyens de sécurité et de protection et éviter toute sources de danger et de menace pour le citoyen, la région et la communauté (https://www. onos.fr) ;

- Les produits dangereux, ne devraient pas être entreposés, transportés ou déplacés dans les collectivités résidentielles, commerciales ou administratives ;

- Les canaux de distribution devraient être faciles, courts, simples et ne pas inclure les intermédiaires ayant un comportement contraire à l'éthique, comme monopoliser la marchandise, contrôler l'offre, augmenter les prix ou utiliser des méthodes inappropriées (https://www.atelier-mb.fr).

- Communication: La communication socialement responsable a les exigences suivantes: l'honnêteté, l'évitement de la fraude et la tromperie, évitement des contestations de biens et de services et modération des dépenses publicitaires, Il faut concevoir des messages positifs et valorisants pour les consommateurs. Et puis, il faut veiller à réduire l'impact écologique de ses communications, enminimisant la

\begin{tabular}{|ll|l|l|}
\hline Volume:04 & $\mathbf{N}^{\circ}: 01$ & Année:2021 & P: 496 \\
& & &
\end{tabular}


consommation de toute forme d'énergie et de papier et bien sûr, favoriser l'utilisation des matières recyclables (Mrani, 2009).

En fin, Si les entreprises ne s'acquittent pas de leurs tâches dans la mise en œuvre de la responsabilité sociale et aider la société pour résoudre les problèmes dont elle souffre, elles peuvent perdre beaucoup de leur influence dans la société et dans le monde des affaires.

\section{3 Initiatives de quelque entreprise en matière de marketing vert et RSE}

Dans cette partie de la recherche on va illustrer par quelques exemples la mise en œuvre habile du marketing vert pour concrétiser la responsabilité sociale de l'entreprise.

- Valneva : Une entreprise leader dans l'industrie du vaccin, né en 2013 de la fusion entre la société française Vivalis et l'entreprise autrichienne Intercell, et dont la mission est de protéger la vie des personnes contre les maladies infectieuses grâce à la médecine préventive. le Groupe Valneva emploie aujourd'hui plus de 400 salariés en France et en Autriche, mais aussi en Ecosse, en Suède, au Canada et aux USA (valneva, 2015).

La pollution de l'environnement par les déchets chimiques étant l'un des problèmes environnementaux les plus importants, la question de la durabilité constitue un défi particulièrement important dans ce secteur.

En 2015, valneva a pris des engagements afin de crédibiliser et donner de la valeur à ses actions et réalisations en matière de responsabilité sociale (normes du travail, normes environnementales, lutte contre la corruption, chaîne d'approvisionnement et impact des produits sur la santé et la sécurité des consommateurs) (valneva, 2015). L'activité de Valneva dépend de sa capacité à fabriquer des vaccins sur ses sites où elle détient $100 \%$ des bonnes pratiques de fabrication. Cette activité présente des risques inhérents (des accidents ou des maladies professionnelles, les effets négatifs sur l'environnement et tout impact sur la sécurité et la qualité de ses produits fabriqués), qu'elle s'efforce de gérer et de prévenir, par: (valneva, 2018)

- Intégration des opérations et les chaînes d'approvisionnement durables, les produits et les emballages innovateurs et la durabilité environnementale dans son processus de prise de décisions et dans ses stratégies marketing;

- la prévention de la pollution, la réduction des déchets et la préservation des ressources naturelles et l'environnement.

Volume:04

$\mathbf{N}^{\circ}: 01$

Année:2021


De plus, le groupe exploite des installations en matière de tri, de recyclage et de suivi des déchets.

- NATURACTIVE: est une marque du groupe pharmaceutique pierre fabre, né en 1988d'après une idéologie basée sur le lien étroit entre la santé et les végétaux (https://www.1001pharmacies.com/naturactive-m209?brands=Naturactive), une marque $100 \%$ responsable elle prend le nom de naturactive en 2009 et compte 12 gammes et 215 références. naturactive utilise des plantes qui n'ont pas d'impact sur la pérennité des ressources et elle est accès sur la phytothérapie, l'aromathérapie et les compléments alimentaires (https://www.easyparapharmacie.com/les-marques-deparapharmacie/naturactive.html).

Afin de satisfaire la demande sans mettre les espèces en danger, Pierre Fabre Agronomie et Naturactive ont entrepris la mise en culture de l'Hélichryse italienne (une plante sauvage qui pousse dans le sud est de la France et plus particulièrement en Corse) en plein champ à Lavaur, dans le Tarn. Elle est aujourd'hui mise en culture dans le souci de sauvegarder l'espèce dans son milieu naturel. Face à la très forte demande en huile essentielle en raison de ses propriétés exceptionnelles pour guérir les blessures et les plaies, les approvisionnements doivent être maitrisées et l'espèce ainsi préservée (il faut distiller $3 \mathrm{~kg}$ de sommités fleuries pour remplir un flacon de 5 $\mathrm{ml}$ ). C'est aussi un moyen de maitriser la production, et de garantir la naturalité, l'efficacité et la sécurité des produits (Naturactive, 2020).

L'adaptation d'une plante dans un nouveau sol est une initiative nouvelle pour le laboratoire Pierre Fabre mais aussi pour les parties prenantes.

Le choix de cultiver cette espèce de plante sur un sol dont les conditions climatiques sont différentes de son terroir d'origine, était un défi pour identifier un itinéraire de culture adapté (pluviométrie plus forte en automne dans le Tarn, sols argileux et non caillouteux). Il a fallu également : (Naturactive, 2020)

- choisir une variété adaptée aux conditions climatiques et aux besoins en qualité d'huile essentielle ;

- mettre en application des outils de récolte adaptés à l'itinéraire de culture élaboré.

Cette démarche a crée une filière contrôlée pour l'hélichryse avec comme avantages: (Naturactive, 2020)

- Un impact contrôlé sur l'environnement (culture issue d'une exploitation certifiée Haute Valeur Environnementale) ;

\begin{tabular}{|ll|l|l|} 
Volume:04 & $\mathrm{N}^{\circ}: 01$ & Année:2021 & P: 498 \\
& & &
\end{tabular}


- $\quad$ une qualité constante et vérifiée de l'huile essentielle ;

- une production locale pilote en cours de déploiement chez des agriculteurs partenaires sous contrat pendant 5 ans, en relation avec la Chambre d'agriculture du Tarn pour fédérer la profession autour de ce nouveau débouché économique (formation, études économiques, réflexions sur le matériel à partager,...).

- Création d'un réseau technique et économique avec les parties prenantes intéressées par la diversification de leur production.

Cette initiative a eu un bel impact sur l'image de la marque avec un taux d'engagement important sur les réseaux sociaux, et en plus de ses hectares le groupe pierre fabre a réussi d'avoir 6 hectares sous contrat avec 3 agriculteurs bio locaux en 2020.

En fin, le groupe implique tous ses services (marketing, juridique, agronomie, communication, ...) pour réussir son projet du marketing vert et investit en permanence dans des stratégies et des domaines de recherche qui peuvent améliorer la durabilité de l'entreprise et sa responsabilité à long terme.

- Le chat (Henkel) : Henkel est une entreprise allemande présente dans trois domaines d'activités, les détergents et l'entretien domestique, les cosmétiques, et les colles et adhésifs, Créé en 1876, Henkel s'appuie sur plus de 140 ans de réussite.Reconnu comme un leader en matière de développement durable et responsabilité sociale, Henkel détient des positions de premier plan dans différents classements et indices internationaux (https://www.henkel.fr/presse-et-medias/dossierspresse/presentation-de-henkel).

Le chat est une marque prise par le groupe allemand Henkel en 1986, et s'est fait connaitre en 1989 par une publicité selon laquelle, elle vantait son produit-vedette le chat-machine (https://fr.wikipedia.org/wiki/Le_Chat_(lessive)).

En avril 2014 la marque le chat a lancé une initiative qui consistait à proposer une solution visant à réduire l'impact sur l'ensemble du cycle de vie avec une offre produit le chat-Eco-Efficacité qui repend au attentes du marché : la performance, car la formule du produit est ultra efficace d'après 60 millions de consommateurs, le respect de l'environnement (un produit éco-conçue et certifié par l'écolabel européen) (https://www.reussir-avec-un-marketing-responsable.org/sites/default/files/lechat lessive-eco-labellisee-eco-efficacite.pdf).

Le Chat Eco Efficacité au Savon d'Origine Végétale, c'est toute l'efficacité Le Chat, avec un impact limité sur l'environnement: (https://www.lechat.fr/fr/accueil/nosproduits/gammes/le-chat-eco-efficacite.html) 
- $\quad$ une lessive certifiée Ecolabel sans compromis sur l'efficacité ;

- $\quad 90 \%$ d'ingrédients d'origine naturelle pour une efficacité pure ;

- $\quad$ une bouteille plus respectueuse de l'environnement contenant $50 \%$ de plastique recyclé et recyclable ;

Le projet a mis un programme de formation des consommateurs aux éco-gestes « Lavons Mieux ! » qui a pour but d'observer les habitudes et préjugés des consommateurs - conçu avec des experts pour répondre simplement et efficacement aux questions des utilisateurs (allergie, économie, écologie) en mêlant expertise et humour, grâce à de courtes vidéos intitulées " Brèves de hublot " (https://www.reussir-avec-un-marketing-responsable.org/sites/default/files/le-chat lessiveeco-labellisee-eco-efficacite.pdf).

Cette démarche a été conçue en collaboration avec l'équipe marketing Le Chat, la recherche et développement, le département RSE, des parties prenantes expertes.

Ce projet a parcouru 3 grandes phases :

Phase1: Conception d'une lessive qui a le pouvoir d'accroitre la cible limitée des consommateurs de lessive écologique: cette phase consiste à démontrer que le chat Eco-Efficacité combine 4 cartes maitresses pour les consommateurs : une marque connue et réconfortante, avec un capital fort dans la santé et l'environnement, efficace même à froid et en cycle court pour réaliser des économies d'énergie et d'eau (https://www.lechat.fr/fr/accueil/nos-produits/gammes/le-chat-eco-efficacite.html) , elle se classe parmi les 7 formules les plus efficaces du marché,un emballage attrayant testé pour satisfaire une cible large,une empreinte environnementale réduite car le produit contient des agents lavant végétales et biodégradable (https://www.reussir-avec-un-marketing-responsable.org/sites/default/files/le-chat lessiveeco-labellisee-eco-efficacite.pdf).

Phase2: Évaluer les habitudes de lavage des consommateurs dans le but d'identifier les pratiques, les connaissances et les préjugés: cette deuxième étape vise à rendre des visites aux consommateurs en les interviewant et en observant les habitudes de lavage, et conclure que les consommateursconnaissent mal l'impact du lavage à ses différentes étapes. Par ailleurs, Ils ignorent les actes responsables efficaces et ont des préjugés importants quant à l'efficacité lavante des lessives écologiques (https://www.reussir-avec-un-marketingresponsable.org/sites/default/files/le-chat lessive-eco-labellisee-eco-efficacite.pdf).

Phase3:laconstruction et la promotion des éco-gestes de lavage efficaces: en finLes observations ont été montrées à des experts (Chimiste, énergéticien, allergologue, 
spécialiste du recyclage, de l'eau ou de la consommation) qui ont ciblé les enjeux majeurs $\mathrm{du}$

lavage(https://www.reussir-avec-un-marketingresponsable.org/sites/default/files/le-chat lessive-eco-labellisee-eco-efficacite.pdf).

Depuis le lancement de cette initiative en 2014, les résultats ont été très bénéfiques pour l'entreprise et pour la société (impact environnemental et social) :(livre blanc, 2016)

- Incidence réduite sur les écosystèmes aquatiques ;

- Usage limité de substances dangereuses ;

- Part de marché doublée ;

- Prix de l'éco-conception en 2014 ;

- Prix «Grandes Entreprises » de la 3ème nuit de la RSE en 2015;

- Projet mobilisateur et formateur sur la RSE qui fait école au sien du groupe

Et pour parvenir à ces résultats encourageants le groupe a entrepris les facteurs de réussite suivant : (livre blanc, 2016)

- Formation des équipes à la RSE et au marketing vert et responsable ;

- Accompagnement des experts ;

- Implication des parties prenantes tout au long du processus ;

- Accepter de travailler sur le long terme.

- L'Oréal: Le groupe L'Oréal, créé en 1909 par Eugène Schueller, est devenu un groupe international numéro un mondial de l'industrie cosmétiques avec de nombreuses marques : Garnier, Lancôme, Maybelline et beaucoup d'autre. Il est dirigé par Jean-Paul Agon. Son actionnaire majoritaire est la famille Bettencourt, descendante du fondateur, représentée par Liliane Bettencourt puis par sa petitefille Françoise Bettencourt-Meyers (https://www.la-croix.com/Economie/loreal-groupeactu-info).

En 2018 de nombreuses marques de L'Oréal (Ushuaïa, L'Oréal Paris, Garnier, Cadum, Narta, Roger\&Gallet, Vichy...) ont décidé de participer à la campagne Trions en beauté, une campagne de sensibilisation au tri des produits de beauté, et pour cela l'oréal etCiteo ont conçu un cite web ( trionsenbeauté.fr ) pour aider le grand public à mieux trier en expliquant comment séparer les emballages des produits d'hygiène et de beauté entre ceux qui vont à la poubelle et ceux qui doivent finir au tri, ainsi que l'importance du recyclage sur notre environnement, et encourage à la mobilisation de tous (https://www.carnetsduluxe.com/business/trions-en-beaute-oreal-incite-tri-produits-

\begin{tabular}{|ll|l|l|} 
Volume:04 & $\mathbf{N}^{\circ}: 01$ & Année:2021 & P: 501 \\
& & &
\end{tabular}


beaute/), et pour se communiquer avec ses consommateurs le groupe a conçu un Bandeau inséré en pub TV, sous le visuel produit qui informe le consommateur sur la recyclabilité du produit.C'est la première fois que les bandeaux du même type que « Mangez cinq fruits et légumes par jour » sont utilisés pour inciter au recyclage.

L'objectif de cette initiative est de : (trions en beauté- L'Oréal, 2019)

- Continuer à montrer le bandeau sur les publicités TV, print et web des marques ;

- Poursuivre la sensibilisation sur le web via des posts sur les réseaux sociaux des marques, à l'aide aussi de vidéo ;

- Déployer la campagne sur les points de vente avec les distributeurs (un projet a eu lieu avec Leclerc, un autre a débuté avec Monoprix, d'autres sont en cours) ;

- Enrichir la plateforme www.trionsenbeaute.fr avec du contenu utile au consommateur.

- Dans 4 ans la moitié du plastique utilisé dans ses emballages sera d'origine recyclée ou bio-sourcée, de plus d'ici à 2025, 100 \% de ses emballages plastiques seront rechargeables, réutilisables, recyclables»

(https://www.carnetsduluxe.com/business/trions-en-beaute-oreal-incite-tri-produitsbeaute/).

Cette initiative a relié la marque avec ses consommateurs, la alimenta d'une action responsable, la rend plus sensible. Le bandeau permet à la marque d'être vue comme plus responsable et engagée auprès des consommateurs.

Dans ce contexte L'Oréal France a réussi à évaluer l'impact que pouvait avoir cette action sur les consommateurs, d'après les résultats de la publicité Elnett diffusait sur des chaines de télévision:(trions en beauté- L'Oréal, 2019)

- $25 \%$ des téléspectateurs se souviennent du bandeau ;

- $\quad 87 \%$ d'entre eux ont l'envie de trier en voyant cette information ;

- $\quad 85 \%$ jugent que ce bandeau met en avant les engagements de la marque ;

- $\quad 96 \%$ estiment que c'est une bonne idée, Dont $64 \%$ estiment que c'est une très bonne idée.

Les résultats de cette action de communication ont été très bénéfiques pour l'entreprise et pour la société (impact environnemental et social):

- soutenir le travail global mis en place par le groupe pour améliorer et maximaliser les emballages qui représentent la moitié de l'impact environnemental des produits, en favorisant l'économie circulaire ; 
- cette initiative a permis d'éviter la consommation de 8705 tonnes de matériaux vierges en 2018 (+19\% vs. 2017) ;

- Le résultat du sondage après visionnage du bandeau démontre que $87 \%$ des personnes sondées déclarent que cela leur donne envie de trier ou recycler leurs produits d'hygiène.

Et pour parvenir à ces résultats concluants et réussir dans son marketing vert impliquant les pratiques de la RSE, le groupe a coordonné les marque et motiver le manager à mobiliser ses équipes; travailler en collaboration avec des jeunes de tous métiers et toutes marques, pour créer les idées; la communication en interne pour transmettre l'initiative et faire en sorte que l'ensemble de l'entreprise se l'emparée, pour ensuite mieux la porter et la défendre (trions en beauté- L'Oréal, 2019).

\section{Conclusion:}

Ces dernières années ont connu de nombreux scandales de corruption sous toutes ses formes éthiques, financières et environnementales, et à mesure que ces abus augmentaient, la pression du gouvernement et de la société civile montait pour Indemniser les dommages causés par les entreprises,Ces pressions ont posé de nouveaux défis aux entreprises en ravivant leur citoyenneté et en sensibilisant les consommateurs lorsqu'ils prennent des décisions d'achat.

Dans ce contexte les entreprises doivent intégrer La démarche de la responsabilité sociale (RSE) dans leur stratégie et dans la conception, la promotion et la commercialisation de l'offre,et doivent renouveler leur approche marketing pour créer des offres correspondant davantage aux enjeux sociétaux et surtout, convaincre les individus de les suivre dans cette transformation.

Sur la base de ce qui précède, nous présentons ci-dessous les résultats de cette recherche :

- $\quad$ Le marketing vert constitue un excellent levier pour créer de la valeur ;

- $\quad$ Les pratiques de la RSE au bénéfice du fonctionnement et du développement durable sont considérées comme des éléments décisifs pour la réussite des entreprises ;

- Les entreprises se montrent plus reconnaissantes de leurs responsabilités à l'égard de l'environnement naturel et des individus, en intégrant le développement durable dans leurs pratiques marketing, et elles démontrent aussi leur capacité à

Volume:04

$\mathrm{N}^{\circ}: 01$

Année:2021 $\mathrm{P}$


mettre en œuvre un marketing qui harmonise leur intérêt et celui de l'environnement et des consommateurs (marketing vert) ;

- $\quad$ Le marketing est une activité majeure à conserver et à améliorer constamment.

Compte tenu de ce qui précède, nous avons formulé les recommandations suivantes :

- Les entreprises devraient intégrer la responsabilité sociale dans leur travail et l'inclure dans les plans et les politiques ;

- $\quad$ Pour que le marketing vert devienne un fait concret, les entreprises doivent penser à leurs utilisateurs, et accorder la priorité au bénéfice de la consommation par un utilisateur d'un produit ou d'un service particulier ;

- $\quad$ Les entreprises doivent s'engager volontairement vis-à-vis du développement durable, si elles veulent adopter une démarche RSE.

\section{Liste Bibliographique:}

\section{- $\quad$ Livre :}

- MALAVAL Philippe, BENAROYA Christophe, (2013), marketing business to business, 5èmme edition, Pearson France, France.

- LAVILLE Elisabeth, (2009), l'entreprise verte, le développement durable change l'entreprise pour changer le monde, 3emme édition, Pearson village mondial édition, France.

- GAY COUTURIER,(2014), guide pratique des marketing, séduire, convaincre et persuader, société des écrivains, France.

- FAHD Rita, (2013) Marketing durable, Vuibert édition, Paris, France.

\section{- $\quad$ Mémoire de fin d'étude et thèses :}

- REMOUSSE N, (2005) le marketing vert, mémoire de master spécialisé en droit de l'environnement, INSA, Lyon, France.

- BERGERON Emilie, (2011), valeur ajoutée et stratégies d'extension en marketing vert, mémoire de maitrise spécialisé en sciences de la gestion, HEC, Montréal, Canada.

- DE GERUS Elsa, (2013), le phénomène du greenwashing et son impact sur les consommateurs, mémoire de maitrise spécialisé en administration des affaires, université du Québec trois rivières, Canada.

- RODIC Ivana, (2007), Responsabilité sociale des entreprises -le développement d'un cadre européen, mémoire d'études approfondies en études européennes, université de Genève, suisse.

- TCHAM Kamel, (2017), Le rôle du marketing dans la protection de l'environnement dans la wilaya d'Oran, thèse Pour l'obtention du diplôme de Doctorat en Sciences,

\begin{tabular}{|ll|l|l|}
\hline Volume:04 & $\mathrm{N}^{\circ}: 01$ & Année:2021 & P: 504 \\
& & &
\end{tabular}


Faculté des Sciences Economiques, Commerciales et des Sciences de Gestion, Université d'Oran 2, Algérie.

\section{- $\quad$ Article du journal :}

- REDJEF Nacéra et ARABA Rabah, (2018), relation entre développement durable et marketing vert, revue des économies nord africains, Chlef, Algérie, vol 14, $\mathrm{n}^{\circ} 19$,

- CHERKAOUI Adil, (2015), La Responsabilité Sociétale des Entreprises : Un Concept Controversé, Revue Economie, Gestion et Société, Université Hassan II Casablanca, $\mathrm{n}^{\circ}$ 3.

- SI TAHAR Amira, MEZIANI Yacine, (2018), La gouvernance de l'entreprise socialement responsable : Cas de la société nationale d'électricité et du gaz (SONELGAZ), Revue Des économies nord Africaines, chlef, Algérie, $\mathrm{Vol} 14, \mathrm{~N}^{\circ} 19$.

- OUBBA Omar, AKRICH Said, (2018), Cadres théoriques d'inspiration de recherches en contrôle de gestion et pilotage de la performance organisationnelle à l'ère de la responsabilité sociétale des entreprises (RSE), International Review of Economics, Management and Law Research, ,2018 , Université Ibn Zohr , Agadir, maroc, vol 01, no 01.

- CHAKOR Abdellatif, DINIA Jihane, (2018), La Responsabilité Sociétale De L'entreprise : Les Différentes Approches Conceptuelles, European Scientific Journal, Vol 14, $\mathrm{N}^{\circ} 10$.

- QUAIREL Françoise, CAPRON Michel, (2013), le couplage responsabilité sociale des entreprises et développement durable : mise en perspective, enjeux et limites, cairn info revue française de socio- économie, France, $\mathrm{N}^{\circ} 11$.

- LOUPPE Albert, (2006), contribution du marketing au développement durable, revue française du marketing, pole universitaire Leonard de Vinci, paris, $\mathrm{N}^{\circ} 208$.

- BENACEUR Fatima Zohra, (2016), Le marketing responsable: une contribution du marketing dans le développement durable, revue Recherches économiques et managériale, université de Biskra- Algérie, $N^{\circ} 19$.

- MAIGNAN Isabelle, (2005), A stakeholder model for implementing social responsibility in marketing, European Journal of Marketing, Vol. 39.

\section{- $\quad$ Article de séminaire :}

MILED Nadia, FARHANI Ramzy, (2012), lorsque le marketing rime avec l'écologie - étude de l'état de la situation et perspectives de développement-, 2emme congres transformare, 1920 mars, paris, France.

\section{- $\quad$ Sites web :}

- $\quad$ www.etudier.com/dissertation/le-marketing-vert, consulté le 04/11/2019.

- www.caderemloi.fr, consulter le 23/12/2020.

- www.generali.fr, consulter le 23/12/2020.

- NOTAT Nicole, STOKKINK Denis, (2010), Responsabilité sociétale des entreprises : la spécificité des sociétés mutuelles dans un contexte européen, Think Tank européen Pour la Solidarité, ,www.pourlasolidarite.eu, consulter le 24/12/2020

- DIMAGGIO. w, POWEL .p, Théorie néo-institutionnelle: isomorphisme et légitimité, dans un champ organisationnel, www.sietmanagement.fr, consulté le 29/12/20.

\begin{tabular}{|ll|l|l}
\hline Volume:04 & $\mathbf{N}^{\circ}: 01$ & Année:2021 & P: 505 \\
& & &
\end{tabular}


- CHAUZAL Christelle,(2007), Réflexion autour du concept de marketing responsable et sur sa déclinaison en termes de communication institutionnelle, http://archives.marketingtrends-congress.com/2007/Materiali/Paper/Fr/Chauzal.pdf, consulter le 23/02/2021

- MRANI Ghizlane, (2009), Contribution de recherche : Marketing sociétal et développement durable, Comment traduire dans son marketing l'engagement sociétal de l'entreprise? Comment mettre en œuvre un marketing et une communication durable,https://visionarymarketing.com/wp-content/uploads/2009/09/cr-v-finale-gmrani.pdf, consulter le 02/03/2021.

- https://www. onos.fr/startupguide/gestion/quest-ce-que-le-marketing-vert, consulter le 05/03/2021.

- https://www.atelier-mb.fr/blog/marketing-ecologique-comment-ladopter, consulter le 05/03/2021.

- Responsabilité societale de l'entreprise valneva, a journey of success, Rapport RSE 2015,www.valneva.com , consulter le 06/03/2021.

- Responsabilité sociétale de l'entreprise, rapport RSE 2018, https://valneva.com/wpcontent/uploads/2019/07/Valneva 2018 Rapport RSE DPEF.pdf, consulter le 05/03/2021.

- https://www.1001pharmacies.com/naturactive-m209?brands=Naturactive, consulter le 08/03/2021.

- https://www.easyparapharmacie.com/les-marques-de-parapharmacie/naturactive.html, consulter le 08/03/2021

- Mise en culture de l'hélichryse bio pour une filière contrôlée, par naturactive,https://www.reussir-avec-un-marketingresponsable.org/sites/default/files/reussir avec un marketing responsable 2020naturactive.pdf,consulter le 08/03/2021.

- https://www.henkel.fr/presse-et-medias/dossiers-presse/presentation-de-henkel, consulter le $08 / 03 / 2021$.

- https://fr.wikipedia.org/wiki/Le Chat (lessive), consulter le 08/03/2021.

- https://www.reussir-avec-un-marketing-responsable.org/sites/default/files/le-chat lessiveeco-labellisee-eco-efficacite.pdf, consulter le 09/03/2021.

- https://www.lechat.fr/fr/accueil/nos-produits/gammes/le-chat-eco-efficacite.html, consulter le $09 / 03 / 2021$.

- https://reussir-avec-un-marketing-responsable.org/sites/default/files/livre-blanc-reussiravec-un-marketing-responsable-2eme-edition.pdf, consulter le 09/03/2021.

- https://www.la-croix.com/Economie/loreal-groupe-actu-info, consulter le 09/03/2021 .

- https://www.carnetsduluxe.com/business/trions-en-beaute-oreal-incite-tri-produits-beaute/, consulter le 10/03/2021.

- https://www.reussir-avec-un-marketingresponsable.org/sites/default/files/trions en beaute loreal - fiches bp 2019.pdf,consulter le $10 / 03 / 2021$. 